\title{
The Effect of Magnetic Field Direction on the Imaging Quality of Scanning Electron Microscope
}

\author{
Libo Ai, Shengxiang Bao*, Yongda Hu, Xueke Wang, and Chuan Luo \\ State Key Laboratory of Electronic Thin Films and Integrated Devices, University of Electronic Science \\ and Technology of China, Jianshe North Street Chengdu 610054, Sichuan, PR China
}

(Received 30 November 2016, Received in final form 18 December 2016, Accepted 19 December 2016)

\begin{abstract}
The significant reduction of the image quality caused by the magnetic field of samples is a major problem affecting the application of SEM (scanning electron microscopy) in the analysis of electronic devices. The main reason for this is that the electron trajectory is deflected by the Lorentz force. The usual solution to this problem is degaussing the sample at high temperatures. However, due to the poor heat resistance of some electronic components, it is imperative to find a method that can reduce the impact of magnetic field on the image quality and is straightforward and easy to operate without destroying the sample. In this paper, the influence of different magnetic field directions on the imaging quality was discussed by combining the experiment and software simulation. The principle of the method was studied, and the best observation direction was obtained.
\end{abstract}

Keywords : SEM, image quality, magnetic field, COMSOL

\section{Introduction}

Since the advent of SEM in the 1960s, it has become an indispensable tool in the analysis of materials and electronic components because of its simple operation and intuitive display.

There are many factors that affect the image quality of the scanning electron microscope. The effect of temperature on the image quality of SEM has been discussed, and the trend of temperature influence on picture quality at a magnification of 100000 times was obtained [1]. Shi et al. [2] found that some of the samples under the action of electron beam react, and thus affect the image quality. Bernstein et al. [3] investigated the effect of SEII on the quality of SEM images of high resolution, and devised a method to reduce its influence. Ko \& Chung [4] studied the effect of charge on the image quality of the insulating material under the electron beam.

Due to the limitations of the principle of scanning electron microscopy, the magnetic field will have a great impact on the image quality and results. Many researchers are working to reduce the effect of magnetic field on the

(C)The Korean Magnetics Society. All rights reserved.

*Corresponding author: Tel: +86-18628299027

Fax: +86-028-83206669, e-mail: sxbao@uestc.edu.cn quality of scanning electron microscopy. Software methods are often used for image signal processing to restore the image, which is distorted by the periodic electromagnetic interference $[5,6]$. Some technicians prefer to perform electromagnetic shielding of the entire SEM laboratory to reduce and eliminate the impact of the electromagnetic field.

However, the above method is generally ineffective when the sample itself is magnetic. The general practice is to degauss the sample at high temperatures, but with the popularity of scanning electron microscopy analysis in the electronics industry, more and more magnetic electronic devices and integration circuit systems need to be analyzed using scanning electron microscopy $[7,8]$. Unfortunately, most of these samples can not undergo high-temperature degaussing treatment. In this paper, the effect of the magnetic field direction of the magnetic sample on the image quality of the scanning electron microscope has been studied.

\section{Samples and Method}

The sample was $\mathrm{NdFeB}$ permanent magnet coated with ceramic film, the size of which was $2.6 \mathrm{~mm} \times 2.4 \mathrm{~mm} \times$ $17 \mathrm{~mm}$, and the remanent direction was the length direction, while the value was $1.05 \mathrm{~T}$. The prepared sample 
A

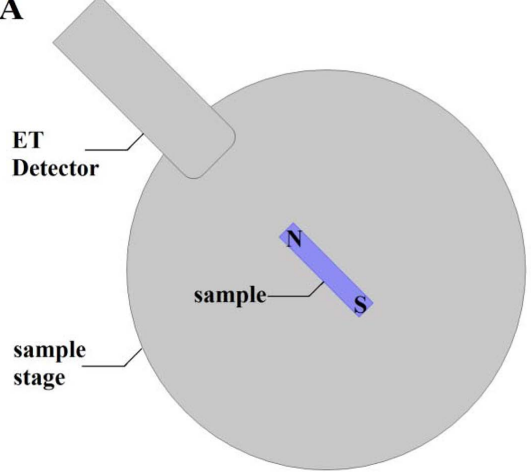

B

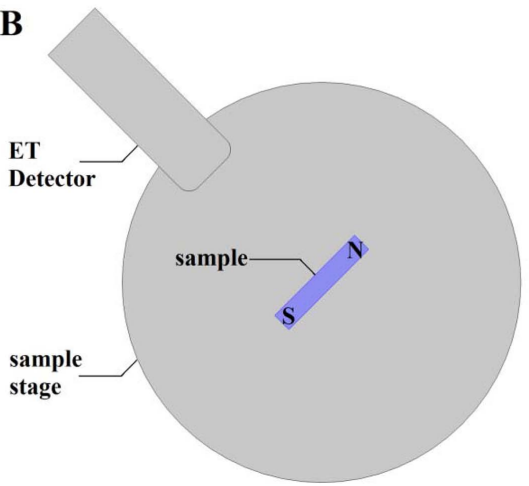

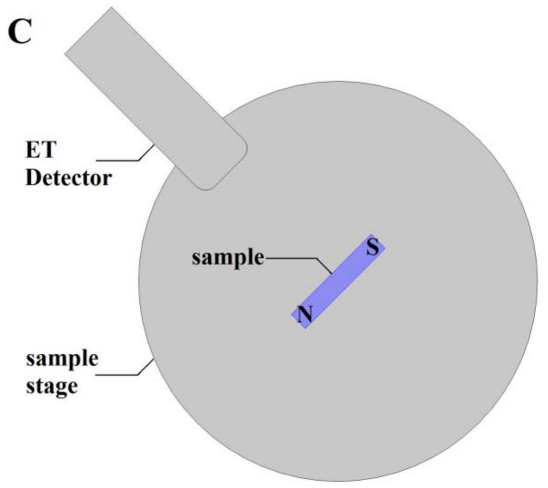

Fig. 1. (Color online) The different direction of the magnetic field of the sample in the observation: (A) the magnetic field direction of the observed region is parallel to the detector, (B) The magnetic field is perpendicular to the detector, (C) the magnetic field is perpendicular to the detector but opposite to the B direction.

was observed using SEM (JSM-6490LV JEOL Tachikawa, Tokyo, Japan) with an electron gun of tungsten filament. As the sample is conductivity poor, to avoid its impact on image quality, the ion sputtering instrument was used to spray gold processing for 15 seconds. As a reference group, one of the samples was mechanically destroyed, and the ceramic film was peeled off and photographed using SEM. The complete sample was placed in the sample chamber in the direction of $\mathrm{A}$, and then the sample stage was rotated in directions B and C, respectively, observed, as shown in Fig. 1.

The WD (working distance) was $10 \mathrm{~mm}$ and the accele- ration voltage was $20 \mathrm{kV}$ during observation, and the brightness and contrast settings of the image were kept the same.

\section{Results and Discussion}

Image 1 in Fig. 2 shows the sample of the ceramic film removed from the magnet. Images 2, 3, and 4 show the complete sample with magnetism corresponding to the magnetic field directions $\mathrm{A}, \mathrm{B}$, and $\mathrm{C}$, respectively.

It can be seen clearly that Image 3 in Fig. 2 is most distinct, and the details of the depressed part are more

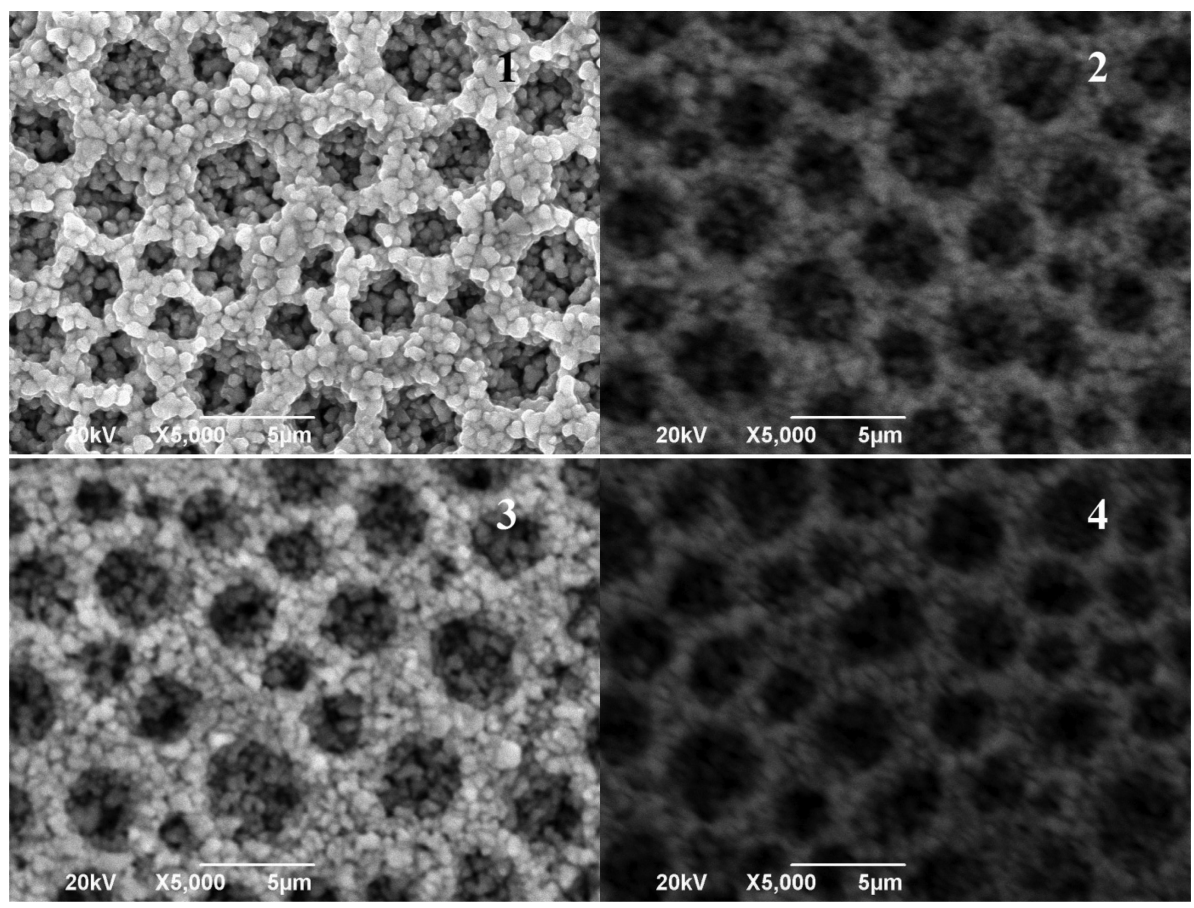

Fig. 2. SEM micrograph of (1) non-magnetic sample, (2) magnetic sample in the direction A, (3) direction B and (4) direction C. 
complete. The resolution of Image 4 is the lowest, and the concave part is very blurred.

SEM image quality is mainly related to its resolution and SNR (signal-to-noise ratio) [9]. The secondary electron image resolution can be derived from the following formula [10]:

$$
\delta=\left(d^{2}+h_{E}^{2}\right)^{\frac{1}{2}}
$$

where $d$ is the effective probe diameter and, $h_{E}$ is the emission depth of the secondary electron. When $d$ is greater than a few nanometers, it can be approximated that $h_{E}$ is equal to $d$. So the above expression can be expressed as [10]:

$$
\delta=\sqrt{2} d
$$

that is the resolution of the picture is mainly determined by the effective probe diameter. In the observation of a magnetic sample, the primary electron beam is affected by the Lorentz force:

$$
F=q(E+v \times B)
$$

where $F$ is the Lorentz force on the electron, $q$ is the charge quantity, $v$ is the electron velocity, $B$ is the magnetic field, and $E$ is the electric field intensity.

Because of the Lorentz force on the primary electron

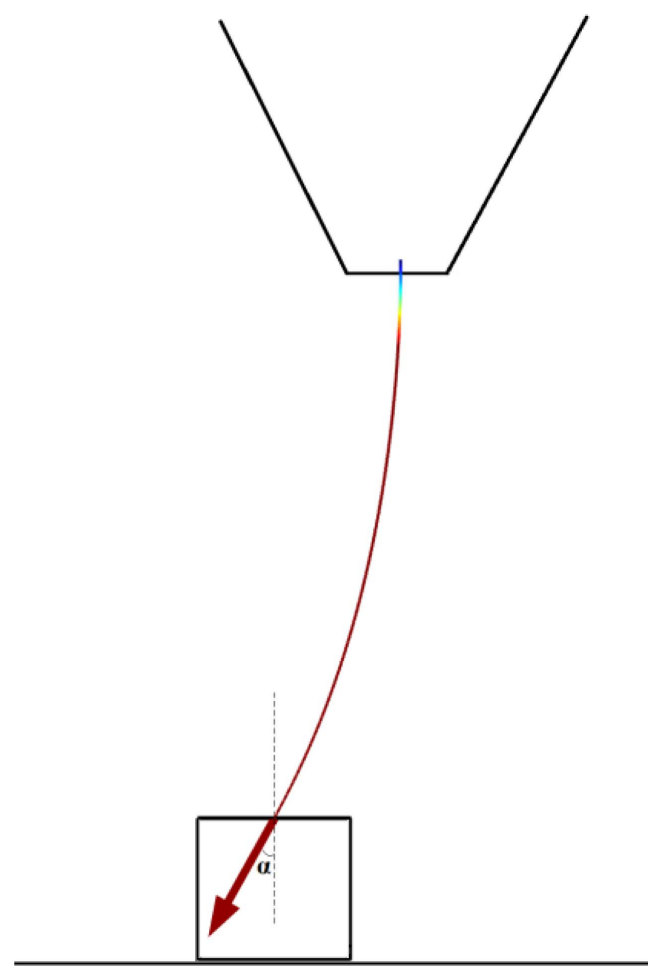

Fig. 3. (Color online) The trajectory (simulated) of the primary electron beam affected by the magnetic field of the sample. beam, the incidence angle would be deflected, and deflection angle $\alpha$, using COMSOL software simulation, can be obtained when the acceleration voltage of $20 \mathrm{kV}, \alpha \approx$ 29.1 ${ }^{\circ}$, as shown in Fig. 3.

For the purpose of this paper a flat sample will be considered since as the primary electron beam is deflected, the effective probe diameter increases, and the shape changes, resulting in astigmatism:

$$
d=d_{0} \sec \alpha
$$

where $d_{0}$ is the original effective probe diameter (not affected by the Lorentz force).

It can be concluded that the deflection of the primary electron beam by the magnetic field led to the increase of the effective probe diameter and reduced the resolution of the image.

SNR is another key factor that affects the quality of secondary electron imaging in scanning electron microscopy.

The empirical formula for the SNR of a SEM image is as follows [11]:

$$
\left(\frac{S}{N}\right)_{\exp }=\left(\frac{I_{p}}{2 e \Delta f}\right)^{\frac{1}{2}}\left(f_{F I} f_{N F-E T}\right)^{\frac{1}{2}}
$$

where $\left(\frac{I_{p}}{2 e \Delta f}\right)^{\frac{1}{2}}$ is the shot noise of the electron probe current, $f_{F I}$ is a function of the frame integration number, and $f_{N F-E T}$ is a function of noise and efficiency of the ET detector (Everhart-Thornley detector) itself, defined as the ratio of the number of pulses at the detector output to the number of incident electrons at the detector input. It is given as:

$$
f_{N F-E T}=\frac{1}{\left(1+\frac{C_{P M T}}{\delta\left(E_{\text {in }}\right)}\right)^{\frac{1}{2}}}
$$

where $C_{P M T}$ is dependent on the relative variance of the photomultiplier multiplication statistics, about 1.5 to 4 , corresponding to the voltage applied to the photomultiplier, and $\delta\left(E_{i n}\right)$ is the number of photoelectrons emitted from the photocathode of the photomultiplier, which is in proportion to the incident electron energy $E_{\text {in }}$ at the surface of the ET detector:

$$
\delta\left(E_{\text {in }}\right) \propto E_{\text {in }}
$$

Therefore, when the $I_{p}$ does not change, SNR has a positive correlation with the incident electron energy $E_{\text {in }}$ at the surface of the ET detector. Because the secondary electron energy is low, $50 \mathrm{eV}$ or less for metal materials, 

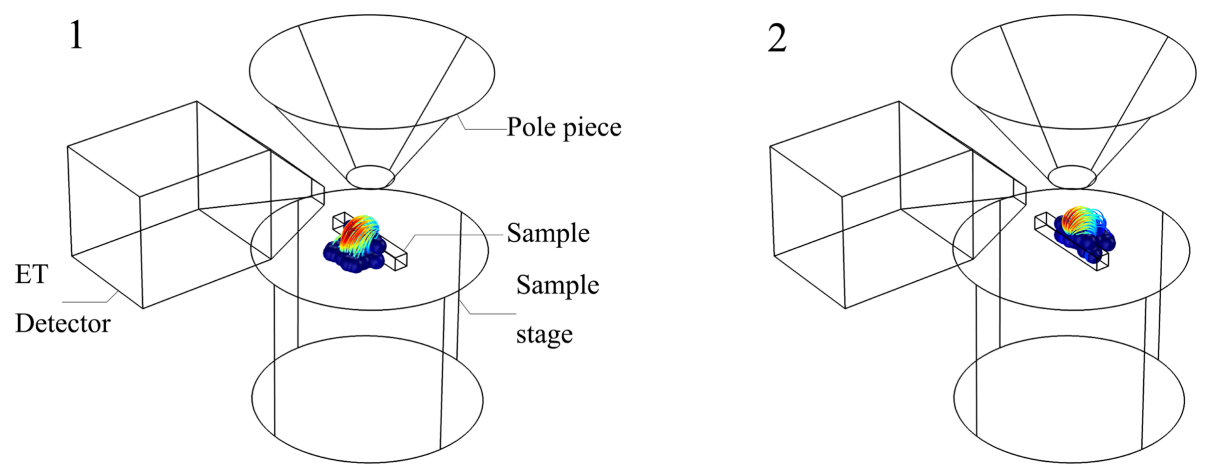

Fig. 4. (Color online) Low - energy secondary electrons trajectories (simulated) in (1) direction B and (2) direction C.

the vast majority of secondary electrons energy is between about 1 and $7 \mathrm{eV}$ [12]. The magnetic field of the sample has a great influence on the SE (secondary electrons) trajectory, so the effect of the different directions of magnetic fields on the $E_{\text {in }}$ is enormous. Moreover, the Faraday cage of ET detector usually applied $+300 \mathrm{~V}$ voltage to attract secondary electrons, thereby increasing collection efficiency, which means the impact of the electric field also needs to be considered. The following Figures (Fig. 4 and Fig. 5) show the influence of magnetic field on the trajectories of secondary electrons with different energy in different magnetic field directions simulated by COMSOL.

It is evident that the secondary electrons were deflected by the Lorentz force in the magnetic field. For low-energy secondary electrons, below $20 \mathrm{eV}$, the magnetic field shielding effect is huge, the electron is almost impossible to be received by the ET detector, regardless of the direction of magnetic field, as shown in Fig. 4. But for high-energy secondary electrons, the energy is about 50 $\mathrm{eV}$, the effect of magnetic field direction on trajectories is enormous, and when the magnetic field was in the $\mathrm{C}$ direction, the electrons were deflected back to the ET detector, and the $E_{\text {in }}$ was significantly reduced. The ET detector was in low efficiency, and the SNR was low,

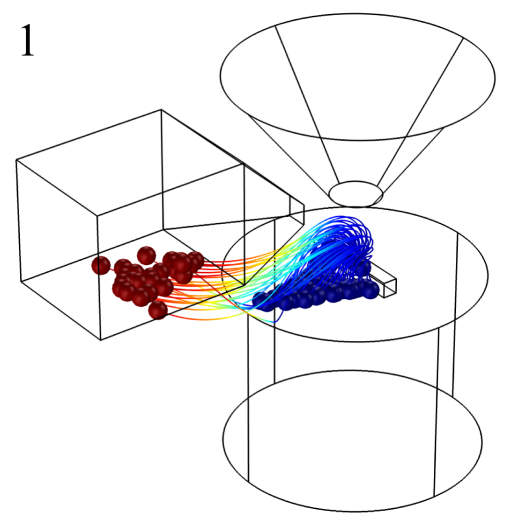

resulting in poor image quality. When the direction of the magnetic field was B, the electrons were deflected toward the ET detector, and the $E_{\text {in }}$ could not change much with respect to the non-magnetic field in the part of highenergy secondary electrons. It is possible to obtain relatively good image quality by observing the sample in the B direction. As shown in Fig. 6.

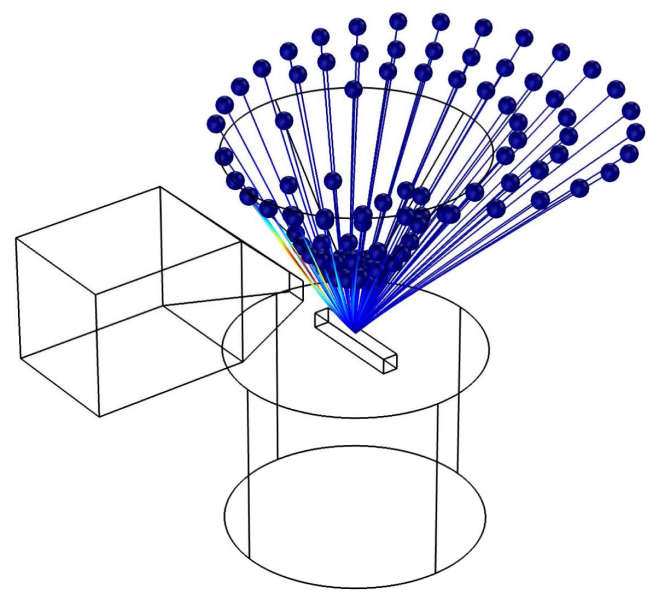

Fig. 6. (Color online) Distribution of backscattered electrons emitted from the sample.

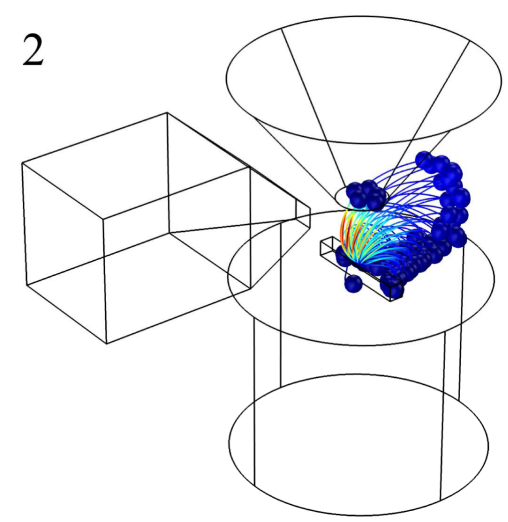

Fig. 5. (Color online) High - energy secondary electrons trajectories (simulated) in (1) direction B and (2) direction C. 


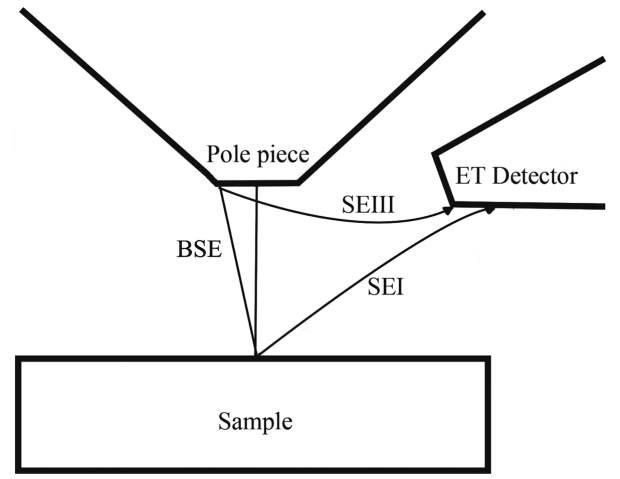

Fig. 7. Trajectories of electrons in the sample chamber.

As for the backscattered electrons, because of their larger energy, similar to the primary electrons, their trajectory is less affected by the electric field on the ET detector [13] and the magnetic field of the sample, as shown in Fig. 6.

SEIIIs are those produced when backscattered electrons emitted from the sample collide with the walls of the sample chamber and the pole piece of the objective lens. The SEIIIs generated from the pole piece account for the majority of SEIIIs' total collection [13], as shown in the following figure (Fig. 7).

SEIIIs contain very limited topographic information [13], so when it is received by the ET detector it would have a negative impact on picture quality, similar to the secondary electrons. The emission energy of SEIII is also low, so the trajectory is also susceptible to sample magnetic field, and the different magnetic field directions on the reception efficiency of the ET detector is also different.

In order to study the influence of the magnetic field on SEIII trajectory and ET detector receiving efficiency, this paper simulated it with COMSOL software, as shown in Fig. 8.
Table 1. Image data for magnets of various directions.

\begin{tabular}{cc}
\hline \hline Direction & SNR \\
\hline A & 3.65 \\
B & 6.67 \\
C & 2.34 \\
\hline
\end{tabular}

It can be seen that SEIIIs were deflected by the Lorentz force. When the direction of the magnetic field was B, the SEIIIs were affected by the Lorentz force, which counteracted some of the electric field force and scattered back to the ET detector. When the direction of the magnetic field was in the $\mathrm{C}$ direction, SEIIIs were influenced by Lorentz force and the electric field force synergistically, so a large number of SEIIIs moved toward to the ET detector, and the number of SEIIIs which were captured by ET detector was much greater than in the B direction. Besides SEIII contains very limited topographic information, in particular at high magnification, a large amount of SEIIIs entered the ET detector, which is equivalent to increasing the noise and further reducing the SNR, which is detrimental to the image quality [13].

Table 1 is measured data for the SNR for control magnetic field directions. The data were obtained using ImageJ software [3]. It can be seen that the results are consistent with the discussion.

\section{Conclusion}

In this work, the effect of different magnetic field directions on the imaging quality is investigated. When the magnetic sample was observed by SEM, the primary electron beam was deflected by the Lorentz force, and the effective probe diameter would be increased, regardless of the direction of the magnetic field, resulting in astigmatism and reducing the final image quality. Because SE and SEIII emission energy are small, their trajectories are
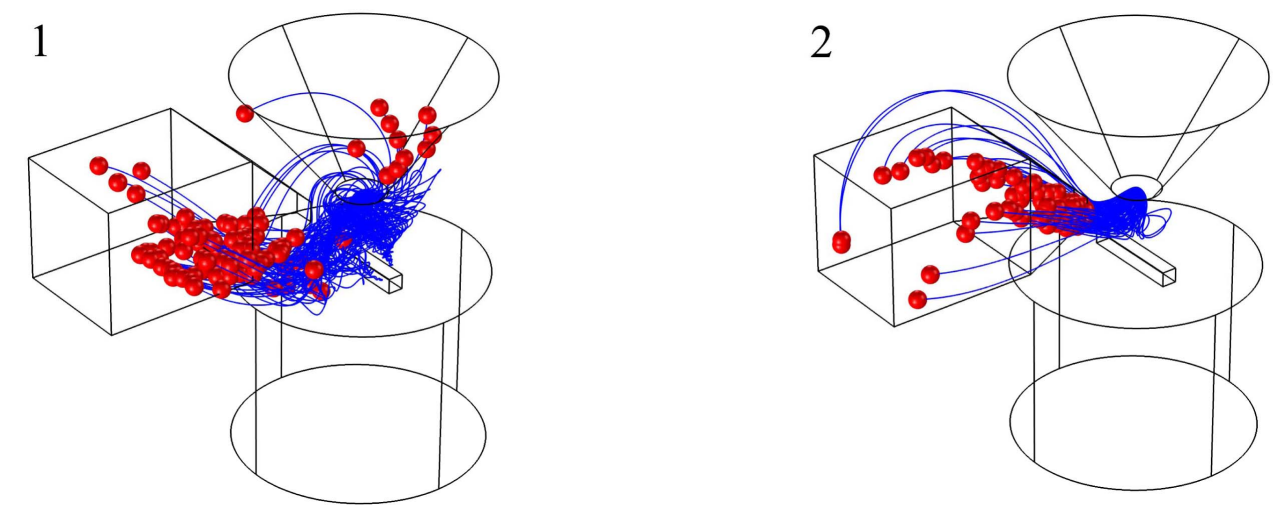

Fig. 8. (Color online) SEIIIs trajectories (simulated) in (1) direction B and (2) direction C. 
influenced by the magnetic field even more. When the sample was observed in the direction $\mathrm{C}$, the SEs were affected by the Lorentz force, and the trajectories were deflected. A large number of SEs moved back toward the ET detector, resulting in the ET detector receiving fewer electrons, and $E_{\text {in }}$ decreasing, while the SEIIIs were heavily biased towards the ET detector. The Noise components were significantly improved, and ultimately greatly reduced the SNR, therefore image quality was very poor. When the direction of the magnetic field is $\mathrm{B}$, the SEs were deflected by the Lorentz force toward the ET detector, $E_{\text {in }}$ was increased, and SEIIIs were deflected toward the ET detector. The Noise component in the signal was reduced, so when the magnetic sample was observed in the B direction, the image quality was much better than in the $\mathrm{C}$ direction. The image quality observed in the direction $\mathrm{A}$ was between in direction B and C. It is expected that the present work may prove beneficial to researchers in the field of electronic device and circuitry failure analysis.

\section{References}

[1] L. Chen and J. Liu, Meas. Sci. Technol. 26, 075402 (2015).

[2] G. Shi, S. Bao, W.Lai, Z. Rao, X. Zang, and Z. Wang, SCANNING. 35, 69 (2013).

[3] G. H. Bernstein, A. D. Carter, and D. C. Joy, SCANNING. 35, 1 (2013).

[4] Y. U. Ko and M. S. Chung, SCANNING. 8, 549 (1998).

[5] M. Pluska, A. Czerwinski, J. Ratajczak, J. Katcki, and R. Rak, J MICROSC. 224, 89 (2006).

[6] M. Pluska, A. Czerwinski, J. Ratajczak, J. Katcki, L. Oskwarek, and R. Rak, Micron. 40, 46 (2009).

[7] H. Fujioka, K. Nakamae, H. Takaoka, and K. Ura, Trans. IECE Japan. E64, 295 (1981).

[8] W. Wan, H. Lu, V. Zhukova, M. Ipatov, A. Zhukov, and Y. Shen, AIP ADV. 6, 095309 (2016).

[9] C. W. Oatley, The Scanning Electron Microscope, Cambridge University Press, London (1972) pp. 84-90.

[10] G. Pfefferkorn, Scanning Electron Microscopy, SEM Inc, AMF O' Hare (1978) pp. 102-105.

[11] T. Agemura, S. Fukuhara, and H. Todokoro, SCANNING. 23, 403 (2001).

[12] H. Seiler, J. Appl. Phys, 54, R1 (1983).

[13] M. Balasubramanyam and E. Munro, Proc. SPIE. 2252, 270 (1995). 